\title{
A NECESSARY CONDITION FOR ANALYTIC HYPOELLIPTICITY
}

\author{
Michael Christ
}

\section{Results}

A partial differential operator, $L$, is said to be analytic hypoelliptic in some open set $\Omega$, if for every open $U \subset \Omega$ and every distribution $u \in \mathcal{D}^{\prime}(U)$, if $L u \in C^{\omega}(U)$, then $u \in C^{\omega}(U)$.

Let $X_{1}, X_{2}$ be real vector fields defined in an open set $\Omega \subset \mathbb{R}^{3}$. Assume them to have analytic coefficients, to be linearly independent at every point, and to satisfy the hypothesis of Hörmander [Ho] that the Lie algebra which they generate spans the tangent space to $\mathbb{R}^{3}$ at each point of $\Omega$. If $L=$ $X_{1}^{2}+X_{2}^{2}$, then $L u \in C^{\infty}(U)$ implies $u \in C^{\infty}(U)[\mathrm{Ho}$.

Fixing any coordinate system, define the determinant

$$
\lambda(x)=\operatorname{det}\left(X_{1}, X_{2},\left[X_{1}, X_{2}\right]\right) .
$$

A point $x \in \mathbb{R}^{3}$ is said to be of type 2 if $\lambda(x) \neq 0$, and otherwise to be a point of higher type. We say that a real curve $\gamma:(-\varepsilon, \varepsilon) \mapsto \mathbb{R}^{3}$ is subordinate to $\left\{X_{1}, X_{2}\right\}$ if $\dot{\gamma}(t) \in \operatorname{span}\left\{X_{1}, X_{2}\right\}$ at $\gamma(t)$, and if $\dot{\gamma}(t) \neq 0$, for all $|t|<\varepsilon$.

We confirm the following special case of a belief expressed by Treves $[\operatorname{Tr}]$.

Theorem 1. $L=X_{1}^{2}+X_{2}^{2}$ is not analytic hypoelliptic in any open set containing a subordinate curve composed only of points of higher type.

A simple corollary is that $L$ cannot be analytic hypoelliptic in any open set in which $\lambda$ changes sign. It is this author's conviction that analytic hypoellipticity can fail, for sums of squares of two vector fields in $\mathbb{R}^{3}$, in other situations besides that of the theorem.

The pair $X_{1}, X_{2}$ defines a CR structure, so that $\bar{\partial}_{b}=X_{1}+i X_{2}$ is a Cauchy-Riemann operator associated to the structure. Denote by $\bar{\partial}_{b}{ }^{*}$ the

Received November 30, 1993.

Research supported by the National Science Foundation. 
formal adjoint of $\bar{\partial}_{b}$, with respect to any nonvanishing real analytic density. The CR structure is said to be pseudoconvex if $\lambda$ never changes sign, and to be of finite type if the bracket condition of Hörmander is satisfied. It is weakly pseudoconvex at those points where $\lambda$ vanishes.

Theorem 2. Suppose that $\bar{\partial}_{b}$ is a Cauchy-Riemann operator associated to a real analytic, pseudoconvex $C R$ structure of finite type in $\Omega \subset \mathbb{R}^{3}$, and that $\Omega$ contains a nonconstant real curve $\gamma$ with

$$
\dot{\gamma}(s) \in \operatorname{span}\left\{\Re\left(\bar{\partial}_{b}\right), \Im\left(\bar{\partial}_{b}\right)\right\}(\gamma(s)),
$$

and such that the $C R$ structure is weakly pseudoconvex at $\gamma(s)$, for all $s$. Then there exists an open subset $U \subset \Omega$ and a function $u \in C^{\infty}(U)$, such that $\bar{\partial}_{b} \bar{\partial}_{b}{ }^{*} u \in C^{\omega}(U)$ but $\bar{\partial}_{b}{ }^{*} u \notin C^{\omega}(U)$.

This kind of regularity is the natural one in the context of $\bar{\partial}_{b}[\mathrm{~K}]$.

Some of the partial results which have been known concerning sufficient and necessary conditions for analytic hypoellipticity of these and related operators are as follows.

(1) $L$ is analytic hypoelliptic in any open set in which $\lambda$ never vanishes [Ta1],[Ta2],[Tr], and likewise for $\bar{\partial}_{b}[\mathrm{G}]$.

(2) In $\mathbb{R}^{d+1}$, a sum $L=\sum X_{j}^{2}$ of squares of $d$ pointwise linearly independent analytic vector fields, satisfying Hörmander's condition, fails to be analytic hypoelliptic in any open set in which the determinant of an associated Levi form, generalizing $\lambda$, vanishes identically $[\mathrm{M}]$. This condition is vacuous, though, for two vector fields in dimension three satisfying the bracket condition, and is not optimal in higher dimensions.

(3) $L$ is analytic hypoelliptic in certain cases in which $\lambda$ vanishes somewhere, including

$$
X_{1}=\partial_{x_{1}}, \quad X_{2}=\partial_{x_{2}}+a\left(x_{1}, x_{2}\right) \partial_{x_{3}}
$$

where $\lambda=\partial_{x_{1}} a=x_{1}^{m}+x_{2}^{n}$ for any even, positive integers $m, n$ [GS]; in this case $\lambda$ vanishes along a curve which, however, fails to be subordinate.

(4) For $m \geq 3, L$ fails to be analytic hypoelliptic for

$$
X_{1}=\partial_{x_{1}}, \quad X_{2}=\partial_{x_{2}}+x_{1}^{m-1} \partial_{x_{3}}
$$

$[\mathrm{He}],[\mathrm{Pr}],[\mathrm{HH}],[\mathrm{C} 1],[\mathrm{C} 2]$; likewise for $\bar{\partial}_{b}$ in the pseudoconvex case of even $m$ [CG],[C3], and more generally for differential operators expressible as (generic) homogeneous polynomials, with constant coefficients, in these two vector fields [C4].

(5) For

$$
X_{1}=\partial_{x_{1}}, \quad X_{2}=\partial_{x_{2}}+\left(x_{1}^{m-1}+x_{1} x_{3}^{M}\right) \partial_{x_{3}},
$$


$\bar{\partial}_{b}$ fails to be analytic hypoelliptic in the sense of Theorem 2, provided that $m, M$ are even and sufficiently large [C5]. These examples were the first, with only a one-dimensional set of points of higher type, in which nonanalytic solutions were proved to exist.

The model vector fields (4) are not merely examples. In Lemma 1, below, it is shown how any structure satisfying the hypotheses of Theorem 1 may be viewed as a small perturbation, along $\gamma$, of one of these model structures. The examples (5) were analyzed by a method that treated them as perturbations of (4); that method had weaknesses which prevented it from succeeding except for suitably small perturbations, whence the hypothesis that $m, M$ be large. Here we outline a modification which renders it both more precise, and simpler. Full details will appear elsewhere.

It is not altogether natural to begin with the three-dimensional case. While the mechanism behind the models (4) and Theorems 1 and 2 does exploit three independent variables, there exist operators $L=X^{2}+Y^{2}$ in $\mathbb{R}^{2}$ which fail to be analytic hypoelliptic, yet are elliptic except at a single point. This matter will be investigated in a forthcoming paper. Rather simple examples demonstrate that higher dimensions give rise to more complex phenomena, hence that our analysis cannot extend in a straightforward manner.

\section{Proof}

A preliminary reduction will help us to view $L$ as a perturbation of model operators (4).

Lemma 1. Suppose that through $x_{0}$ there passes a subordinate curve $\gamma$, which contains only points of higher type. Then there exists an integer $m \geq 3$ such that arbitrarily close to $x_{0}$ may be found an open set $U$, on which there are coordinates $y$ with $0 \in U$, and real vector fields $\tilde{X}_{1}, \tilde{X}_{2}$ on $U$ which are linear combinations, with real analytic coefficients, of $X_{1}, X_{2}$, which are linearly independent at every point of $U$, and which take the form

$$
\tilde{X}_{1}=\partial_{y_{1}}, \quad \tilde{X}_{2}=\partial_{y_{2}}+b(y) \partial_{y_{3}}
$$

where $b \in C^{\omega}$,

$$
\begin{array}{ll}
b(y)=a\left(y_{2}, y_{3}\right) y_{1}^{m-1}+O\left(y_{1}^{m}\right) & \text { if } m \text { is odd } \\
b(y)=a\left(y_{2}, y_{3}\right) y_{1}^{m-1}+O\left(y_{1}^{m}\right)+O\left(y_{1}^{m / 2} y_{3}\right)+O\left(y_{1} y_{3}^{2}\right) & \text { if } m \text { is even }
\end{array}
$$

and where

$$
b\left(0, y_{2}, y_{3}\right) \equiv 0 \quad \text { and } \quad a(0)>0 .
$$


In these coordinates $\lambda(y)=\partial b / \partial y_{1}$ vanishes on the subordinate curve $\tilde{\gamma}=\left\{\left(0, y_{2}, 0\right)\right\}$ (and possibly elsewhere).

Theorems 1 and 2 admit refinements in the scale of Gevrey classes $G^{s}$ : if $m$ is as in Lemma 1, then for each $s<m$, there exists $u$ such that $L u \in C^{\omega}$ in $U$, yet $u$ fails to belong to $G^{s}$. Likewise in Theorem 2, there exists $u$ such that $\bar{\partial}_{b} \bar{\partial}_{b}{ }^{*} u \in C^{\omega}$, yet $\bar{\partial}_{b}{ }^{*} u \notin G^{s}$.

In order to prove Theorem 1 we next seek solutions, or near-solutions, for $L$, of the form $u=e^{\phi} h$, where

$$
\phi=\phi_{\zeta, \lambda}\left(y_{2}, y_{3}\right)=i \lambda^{m} y_{3}-\lambda^{m+1} y_{3}^{2}+i \zeta \lambda y_{2},
$$

$\lambda \in \mathbb{R}^{+}$is a large parameter, and $\zeta \in \Gamma \subset \mathbb{C}$, where $\Gamma$ is a small circle centered at a point $\zeta_{0}$ to be described. Substitute $y=\left(\lambda^{-1} x, y_{2}, y_{3}\right)$, and write $z=\left(y_{2}, y_{3}\right)$. An equation $L\left(e^{\phi} h\right)=e^{\phi} g$ becomes $A \varphi=\psi$, where $\varphi(x, z)=h(\lambda x, z), \psi$ is related to $g$ in the same way modulo multiplication by a factor of $\lambda^{2}$, and

$$
A=A_{\zeta, \lambda}=\sum_{|\beta| \leq 2} \lambda^{|\beta|-2} a_{\beta}\left(\lambda^{-1} x, z\right) P_{\beta}\left(\partial_{x}, Y+E\right)
$$

where

$$
\begin{gathered}
Y=i \zeta+\left(i-2 y_{3} \lambda\right) \tilde{b}, \\
\tilde{b}(x, z)=\lambda^{m-1} b\left(\lambda^{-1} x, z\right), \\
E=\lambda^{-1} \partial_{y_{2}}+\lambda^{-m} \tilde{b} \partial_{y_{3}},
\end{gathered}
$$

and $\beta$ ranges over a finite index set so that the $P_{\beta}$ represent all possible monomials of degrees $|\beta| \leq 2$ in two noncommuting variables.

Let $\varepsilon_{0}>0$ be small, and set

$$
D=\left\{z \in \mathbb{C}^{2}:\left|y_{2}\right|<\varepsilon_{0},\left|y_{3}\right|<\varepsilon_{0} \lambda^{-m / 2}\right\} .
$$

Let $H^{2}(D)$ denote the Hilbert space of all holomorphic functions on $D$ which are in $L^{2}(D)$ with respect to Lebesgue measure, and define bounded operators on it by

$$
\begin{aligned}
& \tilde{\partial}_{y_{2}} \sum_{j, k \geq 0} a_{j k} y_{2}^{j} y_{3}^{k}=\sum_{k} \sum_{j \leq \varepsilon_{1} \lambda} a_{j k} j y_{2}^{j-1} y_{3}^{k}, \\
& \tilde{\partial}_{y_{3}} \sum_{j, k \geq 0} a_{j k} y_{2}^{j} y_{3}^{k}=\sum_{j} \sum_{k \leq \varepsilon_{1} \lambda} a_{j k} y_{2}^{j} k y_{3}^{k-1} .
\end{aligned}
$$


Set

$$
Z=\lambda^{-1} \tilde{\partial}_{y_{2}}+\lambda^{-m} \tilde{b} \tilde{\partial}_{y_{3}}
$$

and replace $A_{\zeta, \lambda}$ by

$$
\tilde{A}_{\zeta, \lambda}=\sum_{|\beta| \leq 2} \lambda^{|\beta|-2} a_{\beta}\left(\lambda^{-1} x, z\right) P_{\beta}\left(\partial_{x}, Y+Z\right) .
$$

In order to disprove analytic hypoellipticity, we aim to construct a oneparameter family of trial functions $g_{\lambda}$, such that $L g_{\lambda}$ and many of its derivatives are relatively small for large $\lambda$, while $g_{\lambda}$ itself is not small, thereby contradicting certain Cauchy estimates (detailed in the second-to-last paragraph below) which are a necessary condition for analytic hypoellipticity. We solve

$$
\tilde{A}_{\zeta, \lambda} f_{\zeta, \lambda}=\psi
$$

where $\psi(x, z)=\exp \left(i \xi x-x^{2 m}\right)$ for a fixed constant $\xi$, independent of $\zeta, \lambda$. Let $L^{\prime}$ denote the operator $L$ represented in the $(x, z)$ coordinates, so that

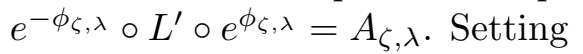

$$
F_{\lambda}=\oint_{\Gamma} e^{\phi_{\zeta, \lambda}} f_{\zeta, \lambda} \zeta^{\sigma} d \zeta
$$

for some nonnegative integer $\sigma$, and noting that $\oint_{\Gamma} e^{\phi_{\zeta, \lambda}} \psi \zeta^{\sigma} d \zeta \equiv 0$,

$$
L^{\prime} F_{\lambda}=\oint_{\Gamma} e^{\phi_{\zeta, \lambda}} A_{\zeta, \lambda} f_{\zeta, \lambda} \zeta^{\sigma} d \zeta=\oint_{\Gamma} e^{\phi_{\zeta, \lambda}}\left(A_{\zeta, \lambda}-\tilde{A}_{\zeta, \lambda}\right) f_{\zeta, \lambda} \zeta^{\sigma} d \zeta
$$

Denoting by $D^{\prime} \Subset D$ the polydisc whose radii are half those of $D$, the operators $\partial_{y_{2}}-\tilde{\partial}_{y_{2}}, \partial_{y_{3}}-\tilde{\partial}_{y_{3}}$ map $H^{2}(D)$ to $H^{2}\left(D^{\prime}\right)$ with operator norms $O(\exp (-c \lambda))$ for some $c>0$, so that this last integrand may be expected to be small for $z \in D^{\prime}$.

The main estimates are as follows.

Lemma 2. There exist $\zeta_{0} \in \mathbb{C}, \xi \in \mathbb{R}, 0 \leq \sigma \in \mathbb{Z}$, a circle $\Gamma$ centered at $\zeta_{0}$, and $\varepsilon_{0}, \varepsilon_{1}, \varepsilon>0$ such that for all sufficiently large $\lambda \in \mathbb{R}^{+}$, there exists for each $\zeta \in \Gamma$ a solution $f_{\zeta, \lambda}$ of

$$
\tilde{A}_{\zeta, \lambda} f_{\zeta, \lambda} \equiv \psi \quad \text { on }\left\{|x|<\lambda^{1 / 2}\right\} \times D
$$

satisfying

$$
\begin{aligned}
& \left|\partial_{x, z}^{\alpha} f_{\zeta, \lambda}(x, z)\right| \leq C^{1+|\alpha|}|\alpha| ! \lambda^{|\alpha| m / 2} e^{-\varepsilon\left|x^{m}\right|} \quad \text { for all } \alpha \\
& \int_{|x| \leq 1}\left|\oint_{\Gamma} e^{\phi_{\zeta, \lambda}} f_{\zeta, \lambda} \zeta^{\sigma} d \zeta\right|^{2} d x \geq c>0 \quad \text { where } z=0 .
\end{aligned}
$$


A consequence is that

$$
\left|\partial^{\alpha}\left(A_{\zeta, \lambda}-\tilde{A}_{\zeta, \lambda}\right) f_{\zeta, \lambda}(x, z)\right| \leq e^{-\varepsilon \lambda} C^{1+|\alpha|}|\alpha| ! \lambda^{|\alpha| m / 2} e^{-\varepsilon\left|x^{m}\right|} \quad \text { for all } \alpha,
$$

for $|x| \leq \lambda^{1 / 2}, z \in D^{\prime}$.

In outline, the proof of the lemma proceeds as follows. Consider

$$
B=\sum_{|\beta|=2} a_{\beta}(0) P_{\beta}\left(\partial_{x}, i \zeta+a(0) x^{m-1}\right),
$$

and denote by $\mathcal{H}^{k}$ the Hilbert space of functions defined on $\mathbb{R}$, and taking values in $H^{2}(D)$, such that

$$
\sum_{0 \leq i+j \leq k} \int_{-\infty}^{\infty}\left\|\left(1+|x|^{m-1}\right)^{i} \partial_{x}^{j} f(x)\right\|_{H^{2}(D)}^{2} e^{\varepsilon_{2}|x|^{m}} d x<\infty .
$$

So long as $\zeta$ avoids a discrete subset of $\mathbb{C}$ and $\varepsilon_{2}$ is chosen to be sufficiently small, $B$ (which acts trivially with respect to $z \in D$ ) defines a bijection of $\mathcal{H}^{2}$ with $\mathcal{H}^{0}$. We modify $a_{\beta}, \tilde{b}$ in such a way that they become defined for all $x \in \mathbb{R}$, but remain unchanged for $|x| \leq \lambda^{1 / 2}$, and show that (if this modification is done properly) the norm of $\tilde{A}_{\zeta, \lambda}-B$, as an operator from $\mathcal{H}^{2}$ to $\mathcal{H}^{0}$, may be made arbitrarily small by choosing $\varepsilon_{0}, \varepsilon_{1}, \varepsilon_{2}$ to be sufficiently small and taking $\lambda$ sufficiently large. A special case of the $L^{2}$ Riesz transform bounds of [RS] is crucial here.

The equation $\tilde{A}_{\zeta, \lambda} f=\psi$ may consequently be solved by Neumann series. There exists $\zeta \in \mathbb{C}$ such that $B_{\zeta}$ has a nonzero solution which is $O\left(\exp \left(-\varepsilon|x|^{m}\right)\right)$ as $x \rightarrow \pm \infty[\mathrm{C} 4]$; fix such a $\zeta_{0}$. $\Gamma$ is a small circle centered at $\zeta_{0}, \sigma$ is chosen so that $\oint_{\Gamma} B_{\zeta}^{-1} \zeta^{\sigma} d \zeta$ is a nonzero operator, and $\xi$ is chosen to ensure that $\psi$ is not in its nullspace.

A delicate point is the splitting of $\partial_{y_{2}}$, for $\tilde{\partial}_{y_{2}}$ must be $\ll \lambda$ in order that the Neumann series be convergent, while $\partial_{y_{2}}-\tilde{\partial}_{y_{2}}$ must map $H^{2}(D)$ to $H^{2}\left(D^{\prime}\right)$ with bound $O(\exp (-c \lambda))$, in order that $\left(A_{\zeta, \lambda}-\tilde{A}_{\zeta, \lambda}\right) f_{\zeta, \lambda}$ will be sufficiently small for the a priori Cauchy estimates in the second-to-last paragraph below to be violated. There is no room to spare.

Return to the coordinates $y$ and define trial functions by

$$
g_{\lambda}(y)=F_{\lambda}\left(\lambda y_{1}, y_{2}, y_{3}\right) \eta_{\lambda}\left(\lambda^{1 / 2} \varepsilon_{0}^{-1} y_{1}\right) \eta_{\lambda}\left(\lambda^{m / 2} \varepsilon_{0}^{-1} y_{3}\right)
$$

where $\eta_{\lambda} \in C_{0}^{\infty}(\mathbb{R})$ is supported in $[-1,1]$, is identically equal to one on $[-1 / 2,1 / 2]$, and satisfies

$$
\left\|d^{k} \eta_{\lambda} / d s^{k}\right\|_{\infty} \leq C^{k+1} \lambda^{k} \quad \text { for all } k \leq \lambda,
$$


with $C$ independent of $\lambda$. The $g_{\lambda}$ are then smooth functions in a fixed open set, independent of $\lambda$. Note that the factor $F_{\lambda}(\cdot)$ is $O(\exp (-c \lambda))$ on the supports of the gradients of the two cutoff functions, because of the rapid $x$-decay in the bounds of Lemma 2 and the factor $\exp \left(-\lambda^{m+1} y_{3}^{2}\right)$ in the definition of $\phi_{\zeta, \lambda}$; this ensures that $L g_{\lambda}$ is still small despite the introduction of the cutoff factors.

The following is a straightforward consequence of Lemma 2, the definitions, and a minor additional calculation.

Lemma 3. There exist $c, \varepsilon>0, C<\infty$, and $p<\infty$ such that for all large $\lambda$ and all $k \leq \lambda$,

$$
\int_{\lambda^{1 / 2}\left|y_{1}\right|+\lambda\left|y_{2}\right|+\lambda^{m}\left|y_{3}\right| \leq 1}\left|\frac{\partial^{k} g_{\lambda}}{\partial y_{3}^{k}}\right|^{2} d y \geq c^{k+1} \lambda^{-p} \lambda^{2 m k}
$$

and such that for $\left|y_{2}\right|<\varepsilon_{0} / 4$, for all $|\alpha| \leq \lambda$,

$$
\left|\partial_{y}^{\alpha} \operatorname{Lg}_{\lambda}(y)\right| \leq C^{|\alpha|+1} \lambda^{m|\alpha|} e^{-\varepsilon \lambda},
$$

and

$$
\left|g_{\lambda}(y)\right| \leq C
$$

A theorem of Métivier [M] asserts that (under a small additional hypothesis, which $L$ does satisfy) if $L$ were analytic hypoelliptic in $\Omega$, then for any open $U^{\prime} \Subset \Omega$ and any compact $K \subset U^{\prime}$, there would exist $C<\infty$ such that for any $g \in C^{\infty}\left(U^{\prime}\right)$, for any $n \in \mathbb{Z}^{+}$,

$\sum_{|\beta|=n}\left\|\partial^{\beta} g\right\|_{L^{2}(K)} \leq C^{n+1} n^{n} \sum_{0 \leq|\alpha| \leq n}|\alpha|^{-|\alpha|}\left\|\partial^{\alpha} L g\right\|_{L^{2}\left(U^{\prime}\right)}+C^{n+1} n^{n}\|g\|_{L^{2}\left(U^{\prime}\right)}$

This criterion opens the door to the use of trial functions for which $L g \notin C^{\omega}$ to disprove analytic hypoellipticity. Let $U$ be as in Lemma 1 and choose $U^{\prime} \subset U$ to be so small a neighborhood of 0 that for $y \in U^{\prime},\left|\exp \left(i \zeta \lambda y_{2}\right)\right| \ll$ $\exp (\varepsilon \lambda)$, uniformly for all $\zeta \in \Gamma$, where $\varepsilon$ is the constant appearing in the second estimate of Lemma 3. Taking $g=g_{\lambda}$, choosing $n \approx \lambda / \sqrt{\log \lambda}$ and exploiting the factor of $\exp (-\varepsilon \lambda)$, one finds that these inequalities do not hold, for $\partial^{n} g_{\lambda} / \partial y_{3}^{n}$, for any finite $C$ independent of $\lambda$, as $\lambda \rightarrow \infty$.

The proof of Theorem 2 proceeds along the same lines, but Métivier's criterion must be adapted to cope with the nonstandard notion of regularity at issue for $\bar{\partial}_{b}$. This amounts to a straightforward microlocalization, and is Proposition 7.1 of [C5]. 


\section{References}

[C1] M. Christ, Some non-analytic-hypoelliptic sums of squares of vector fields, Bulletin AMS 16 (1992), 137-140.

[C2] Certain sums of squares of vector fields fail to be analytic hypoelliptic, Comm. Partial Differential Equations 16 (1991), 1695-1707.

[C3] - Analytic hypoellipticity breaks down for weakly pseudoconvex Reinhardt domains, International Math. Research Notices 1 (1991), 31-40.

[C4] Analytic hypoellipticity, representations of nilpotent groups, and a nonlinear eigenvalue problem, Duke Math. J. 72 (1993), 595-639.

[C5] Examples of analytic nonhypoellipticity of $\bar{\partial}_{b}$, Comm. Partial Differential Equations (to appear).

[CG] M. Christ and D. Geller, Counterexamples to analytic hypoellipticity for domains of finite type, Annals of Math. 235 (1992), 551-566.

[G] D. Geller, Analytic Pseudodifferential Operators For The Heisenberg Group And Local Solvability, Mathematical Notes 37, Princeton University Press, Princeton, NJ, 1990.

[GS] A. Grigis and J. Sjöstrand, Front d'onde analytique et sommes de carrés de champs de vecteurs, Duke Math. J. 52 (1985), 35-51.

$[\mathrm{HH}] \quad$ N. Hanges and A. A. Himonas, Singular solutions for sums of squares of vector fields, Comm. Partial Differential Equations 16 (1991), 1503-1511.

[He] B. Helffer, Conditions nécessaires d'hypoanalyticité pour des opérateurs invariants à gauche homogènes sur un groupe nilpotent gradué, J. Diff. Eq. 44 (1982), 460-481.

[Ho] L. Hörmander, Hypoelliptic second order differential equations, Acta Math. 119 (1967), 147-171.

[K] J. J. Kohn, Estimates for $\bar{\partial}_{b}$ on pseudoconvex $C R$ manifolds, Proc. Symp. Pure Math. 43 (1985), 207-217.

[M] G. Métivier, Une classe d'opérateurs non hypoélliptiques analytiques, Indiana Math. J. 29 (1980), 823-860.

[PR] Pham The Lai and D. Robert, Sur un problème aux valeurs propres non linéaire, Israel J. Math. 36 (1980), 169-186.

[RS] L. P. Rothschild and E. M. Stein, Hypoelliptic differential operators and nilpotent groups, Acta Math. 137 (1976), 247-320.

[Ta1] D. Tartakoff, Local analytic hypoellipticity for $\square_{b}$ on non-degenerate CauchyRiemann manifolds, Proc. Nat. Acad. Sci. USA 75 (1978), 3027-3028.

[Ta2] On the local real analyticity of solutions to $\square_{b}$ and the $\bar{\partial}$-Neumann problem, Acta Math. 145 (1980), 117-204.

[Tr] F. Treves, Analytic hypo-ellipticity of a class of pseudodifferential operators with double characteristics and applications to the $\bar{\partial}-$ Neumann problem, Comm. Partial Differential Equations 3 (1978), 475-642.

Department of M athematics, UCLA, Los Angeles, CA. 90024

E-mail address: christ@math.ucla.edu 